\title{
The magnetic transition, heat capacity, and thermodynamic properties of uranium dioxide from 5 to $350 \mathrm{~K} \uparrow$
}

\author{
JAMES J. HUNTZICKER $\$$ and EDGAR F. WESTRUM, Jr. \\ Department of Chemistry, University of Michigan, \\ Ann Arbor, Michigan 48104, U.S.A.
}

(Received 20 July 1970)

\begin{abstract}
The heat capacity of high purity uranium dioxide has been re-examined over the lowtemperature range especially in the vicinity of the $\lambda$-type anomaly reported to occur at $28.7 \mathrm{~K}$ as a consequence of magnetic ordering. The present study indicates a very sharp transition at $30.44 \mathrm{~K}$ with a maximum $C_{p}$ of $391 \mathrm{cal} \mathrm{mol}^{-1} \mathrm{~K}^{-1}$. The heat capacity at constant pressure $C_{p}$, the entropy $S^{\circ}$, the enthalpy $\left(H^{\circ}-H_{0}^{\circ}\right)$, and Gibbs energy function $\left(G^{\circ}-H^{\circ}\right) / T$ are tabulated and at $298.15 \mathrm{~K}$ have the following values: $15.20 \mathrm{cal} \mathrm{mol}^{-1} \mathrm{~K}^{-1}, 18.41 \mathrm{cal}$ $\mathrm{mol}^{-1} \mathrm{~K}^{-1}, 2696 \mathrm{cal} \mathrm{mol}^{-1} \mathrm{~K}^{-1}$, and $-9.37 \mathrm{cal} \mathrm{mol}^{-1} \mathrm{~K}^{-1}$. The heat capacity results are compared with those derived from the neutron scattering work and also with the theory of Allen.
\end{abstract}

\section{Introduction}

Although the importance of $\mathrm{UO}_{2}$ in nuclear technology has been self-evident, it is only recently that this substance has come under the close scrutiny of solid-state physicists. Much of the increased interest concerns a striking magnetic-ordering transition in $\mathrm{UO}_{2}$ near $30 \mathrm{~K}$. This transition was first detected in thermal capacity measurements by Jones, Gordon, and Long, ${ }^{(1)}$ who found a relatively blunt, $\lambda$-type transformation at $28.7 \mathrm{~K}$ and suggested that the thermal anomaly was associated with a transition from a high-temperature paramagnetic state to a lowtemperature anti-ferromagnetic state. This suggestion has been supported both by magnetic susceptibility studies ${ }^{(2-5)}$ and by powder neutron diffraction measurements. ${ }^{(6)}$

A preliminary report of this research in March $1964^{(7)}$ established that the heat capacities at the peak of the transition were at least 50 times as high as those previously reported and that the transition was a cooperative first-order one rather than a $\lambda$-type anomaly. The Néel temperature $T_{\mathrm{N}}$ ascertained was $30.44 \mathrm{~K}$ or 6 per cent higher than previous reports. These results were corroborated by a detailed diffraction study of the temperature dependence of the antiferromagnetism in single crystal $\mathrm{UO}_{2}$ reported in 1965 by Frazer et al. ${ }^{(8)}$ They found that $T_{\mathrm{N}}$ was $30.80 \mathrm{~K}$ and that 50 per cent of the saturation magnetization was attained within $0.03 \mathrm{~K}$ below $T_{\mathrm{N}}$,

$\dagger$ Supported in part by the Division of Research of the United States Atomic Energy Commission. \$ Present Address: I Physikalisches Institut der Freien Universität Berlin, Berlin, Germany. 
and concluded that the transition was first order by the absence of critical scattering at $T_{\mathbf{N}}$. Similar results were subsequently obtained independently by Willis and Taylor ${ }^{(9)}$ who found a transition temperature of $30.6 \mathrm{~K}$. Both sets of results accord well with thermal results. ${ }^{(7)}$ Anomalies in the elastic constants, ultrasonic attenuation, and thermal expansion were found by Brandt and Walker ${ }^{(10,11)}$ at $T_{\mathrm{N}}$. The anomalous behavior in $c_{44}$ was found to persist to room temperature, whereas $\left(c_{11}-c_{12}\right)$ exhibited smaller and narrower anomalies at $T_{\mathrm{N}}$. In addition, they found a volume change at $T_{\mathrm{N}}$ of 60 to 90 p.p.m. This volume change was confirmed by de Kouchkovsky and Lecomte $^{(12)}$ in an X-ray diffraction study. No lowering of the cubic symmetry was observed, however. Aring and Sievers ${ }^{(13)}$ measured the temperature dependence of the thermal conductivity in $\mathrm{UO}_{2}$ and found a large decrease with a minimum at $T_{\mathrm{N}}$. This depression was by no means sharp, but was extended over a range of almost $200 \mathrm{~K}$.

In addition to the work mentioned above, which dealt primarily with the transitional properties of $\mathrm{UO}_{2}$, very extensive neutron diffraction studies were made both above and below the Néel temperature. Dolling, Cowley, and Woods ${ }^{(14)}$ investigated the crystal dynamics of $\mathrm{UO}_{2}$ at room temperature by inelastic neutron scattering. They interpreted their results in terms of an interionic force model, which took into account the ionic polarizabilities, and derived various thermodynamic quantities, including the harmonic lattice contribution to $C_{V}$. Detailed measurements of the magnetic excitations in the antiferromagnetic phase made by Dolling and Cowley ${ }^{(15,16)}$ included the dispersion relations for the magnon modes below $T_{\mathrm{N}}$. They interpreted their results in terms of a spin-wave excitation Hamiltonian with eight variable parameters. From the model which best fits the experimental results, Cracknell and Joshua ${ }^{(17)}$ calculated the density of states and the magnon contribution to $C_{V}$ in the temperature range 4 to $20 \mathrm{~K}$. Dolling and Cowley ${ }^{(18)}$ have also demonstrated the existence of a strong interaction between the magnons and phonons in the antiferromagnetic state. They have observed that the phonon dispersion relationships are drastically different at 9 and $90 \mathrm{~K}$.

In the course of a series of investigations on the heat capacity of stable and metastable uranium oxide compositions by Westrum et al. , $^{(19-22)}$ the presence of a small $\left[\Delta S_{\mathrm{t}}=0.09 \mathrm{cal} \mathrm{mol}^{-1} \mathrm{~K}^{-1}\right]$ transition in $\alpha-\mathrm{U}_{3} \mathrm{O}_{7}$ at $30.5 \mathrm{~K}$ was revealed. ${ }^{(19)}$ Since this metastable form was prepared by oxidation of $\mathrm{UO}_{2}$ at 50 to $135^{\circ} \mathrm{C}$, the probability of residual $\mathrm{UO}_{2}$ could not be excluded. ${ }^{(20,21)}$ Failure of the observed transition to coincide in temperature with that reported by Jones et al. was without explanation since solid solution formation between $\mathrm{UO}_{2}$ and $\mathrm{U}_{3} \mathrm{O}_{7}$ was not anticipated. The reported analysis ( 99.3 moles per cent of $\mathrm{UO}_{2}, 0.7$ moles per cent of $\mathrm{UO}_{3}$ ) of the Jones et al. ${ }^{(1)}$ sample could probably be better interpreted in terms of about 3 moles per cent of $\mathrm{UO}_{2.25}$ as impurity on the basis of the phase diagram by Grønvold. ${ }^{(23)}$ Hence their sample was so impure as to cause concern about the reliability of the transition temperature and shape of the $C_{p}$ curve. Because of the unexplained thermal anomaly in $\alpha-\mathrm{U}_{3} \mathrm{O}_{7}$, the importance of $\mathrm{UO}_{2}$ in nuclear technology, the key role of $\mathrm{UO}_{2}$ in actinide thermodynamics, and the rather unusual shape reported for the heat capacity of $\mathrm{UO}_{2}$ in the transition region, further study of the thermal properties on two well-characterized $\mathrm{UO}_{2}$ samples was undertaken. 
Our calorimetric sample had better defined phase and chemical composition than that of Jones et al. ${ }^{(1)}$ and thus our results more accurately reflect the thermal properties of $\mathrm{UO}_{2}$. In section 2 the experimental technique is described and in section 3 the numerical results are presented. In section 4 these results are discussed and compared with other experimental work and, where possible, correlated with the predictions of various theories formulated to explain the unusual behavior of $\mathrm{UO}_{2}$.

\section{Experimental}

CRYOSTAT AND CALORIMETER

The Mark II adiabatic cryostat and the technique employed have been described previously. ${ }^{(24)}$ The gold-plated copper calorimeter (laboratory designation W-31) has a capacity of $50 \mathrm{~cm}^{3}$ and six radial vanes. The heat capacity of the calorimeterheater-thermometer assembly, which typically ranged from 22 to a maximum of 30 per cent of the total observed, was determined separately, and adjustments were applied for minute differences in the amounts of Cerroseal (In $+\mathrm{Sn}$ ) solder and Apiezon-T grease employed. A capsule-type, strain-free, platinum-resistance thermometer $(\mathrm{A}-5)$, contained within an entrant well in the calorimeter, is considered to reproduce the thermodynamic temperature scale to within $0.07 \mathrm{~K}$ from 5 to $10 \mathrm{~K}$, to within $0.03 \mathrm{~K}$ from 10 to $90 \mathrm{~K}$, and to within $0.04 \mathrm{~K}$ above this temperature. Precision is considerably better and temperature increments are probably reliable to within $0.001 \mathrm{~K}$ after correction for quasi-adiabatic drift. All measurements of resistance, potential, temperature, time, and mass are referred to calibrations made by the National Bureau of Standards.

The calorimeter contained a sample of mass $274.0126 \mathrm{~g}$, and helium gas at 50 Torr and $25^{\circ} \mathrm{C}$ was added to the sample space after evacuation to facilitate thermal equilibration.

\section{CHARACTERIZATION OF SAMPLES}

A sample of $\mathrm{UO}_{2}$ was provided through the offices of E. D. North of Mallinckrodt Nuclear Corporation and F. M. Belmore of Mallinckrodt Chemical Works. This material was in the form of cylindrical pellets $(1 \mathrm{~cm}$ in diameter and $2 \mathrm{~cm}$ in length) made by pressing $\mathrm{UO}_{2}$ powder to a "green" density of $6.5 \mathrm{~g} \mathrm{~cm}^{-3}$ and firing in a hydrogen atmosphere at temperatures up to $1925 \mathrm{~K}$. Metallic impurities detected by spectrochemical analyses were: $\mathrm{B}, 0.5 ; \mathrm{Cr}, 10 ; \mathrm{Cu}, 0.3 ; \mathrm{Fe}, 60 ; \mathrm{Ni}, 150 ; \mathrm{Pb},<1$; $\mathrm{Si}, 100 ; \mathrm{Sn},<1$ p.p.m. by mass. After adjustment of the oxygen content to slight hyperstoichiometry $(\mathrm{O} / \mathrm{U}=2.002 \pm 0.001)$, chemical analysis indicated $(11.87 \pm 0.01)$ mass per cent of oxygen (theoretical value: 11.85$)$ and uranium $(88.2 \pm 0.17)$ mass per cent (theoretical value: 88.15). The measurements described in this paper are based upon this sample.

Measurements were also made on a second $\mathrm{UO}_{2}$ sample prepared for this study by the Uranium Division of Mallinckrodt through the cooperation of C. W. Kuhlman. This sample was in the form of elongated, flame-fused boules of mass 30 to $110 \mathrm{~g}$. Standard chemical gravimetric analysis indicated the oxygen to uranium mole ratio 
to be $2.000 \pm 0.001$. For unexplained reasons the magnitude of the entropy increment associated with the transition was less than 75 per cent of that observed on the former sample and the broader, hump-shaped, lower heat capacity curve and depressed transition temperature (maximum at $30.07 \mathrm{~K}$ ) led to the rejection of the results from this sample. These rejected experimental results are, however, detailed and available elsewhere ${ }^{(25)}$ with adjuvant values and correlations.

TABLE 1. Heat capacity at constant pressure for uranium dioxide

\begin{tabular}{|c|c|c|c|c|c|}
\hline$\underline{T}$ & $C_{p}$ & $\underline{T}$ & $C_{p}$ & $T$ & $C_{p}$ \\
\hline$\overline{\mathbf{K}}$ & $\mathrm{cal} \mathrm{K} \mathrm{K}^{-1} \mathrm{~mol}^{-1}$ & $\overline{\mathrm{K}}$ & cal K $\mathrm{K}^{-1} \mathrm{~mol}^{-1}$ & $\overline{\mathbf{K}}$ & cal K ${ }^{-1} \mathrm{~mol}^{-1}$ \\
\hline \multicolumn{2}{|c|}{ Series I } & 7.98 & 0.0263 & 30.446 & 197.71 \\
\hline 87.57 & 5.982 & 8.97 & 0.0424 & 30.45 & 160.11 \\
\hline 92.88 & 6.344 & 10.10 & 0.0676 & 30.68 & 2.881 \\
\hline 95.94 & 6.552 & 11.37 & 0.0995 & 31.17 & 2.299 \\
\hline 103.51 & 7.061 & 12.74 & 0.1504 & 31.98 & 2.284 \\
\hline 111.31 & 7.591 & 14.16 & 0.2241 & & \\
\hline 119.28 & 8.116 & 15.64 & 0.3260 & \multirow{2}{*}{\multicolumn{2}{|c|}{ Series III }} \\
\hline 123.16 & 8.368 & 17.13 & 0.4575 & & \\
\hline 130.93 & 8.868 & 18.63 & 0.6234 & \multicolumn{2}{|c|}{ Enthalpy Run A } \\
\hline 139.10 & 9.359 & 20.21 & 0.8380 & 17.00 & 0.5055 \\
\hline 147.91 & 9.855 & 21.64 & 1.0736 & 22.16 & 1.2045 \\
\hline 156.82 & 10.362 & 22.69 & 1.2771 & 26.50 & 2.374 \\
\hline 165.32 & 10.790 & 23.51 & 1.4542 & 28.488 & 3.402 \\
\hline 173.85 & 11.204 & 24.18 & 1.618 & 29.51 & 4.658 \\
\hline 182.97 & 11.621 & 24.78 & 1.776 & 30.17 & 7.035 \\
\hline 192.23 & 12.018 & 25.35 & 1.942 & 30.37 & 24.35 \\
\hline 200.97 & 12.362 & 25.90 & 2.124 & 30.43 & $\begin{array}{r}24.53 \\
385.50\end{array}$ \\
\hline 209.51 & 12.669 & 26.44 & 2.316 & $\begin{array}{l}30.43 \\
30.70\end{array}$ & $\begin{array}{r}385.30 \\
4.664\end{array}$ \\
\hline 217.84 & 12.967 & 26.95 & 2.527 & $\begin{array}{l}30.70 \\
31.79\end{array}$ & $\begin{array}{l}4.004 \\
2.290\end{array}$ \\
\hline 226.06 & 13.256 & 27.44 & 2.752 & & \\
\hline 234.09 & 13.499 & 27.90 & 3.001 & \multirow{2}{*}{\multicolumn{2}{|c|}{ Series IV }} \\
\hline 242.18 & 13.763 & 28.22 & 3.179 & & \\
\hline 250.30 & 13.992 & 28.42 & 3.345 & \multirow{2}{*}{\multicolumn{2}{|c|}{$\begin{array}{c}\text { Series IV } \\
\Delta H_{\mathrm{t}} \text { Run B }\end{array}$}} \\
\hline 258.56 & 14.236 & 28.61 & 3.489 & & \\
\hline 266,82 & 14.455 & 28.80 & 3.638 & 33.94 & 2.323 \\
\hline 275.04 & 14.656 & 28.97 & 3.823 & 36.56 & 2.437 \\
\hline 283.24 & $\begin{array}{l}14.050 \\
14.855\end{array}$ & 29.14 & 4.003 & 40.07 & 2.630 \\
\hline 291.33 & $\begin{array}{l}14.053 \\
15.04\end{array}$ & 29.34 & 4.281 & 44.68 & 2.918 \\
\hline 299.33 & 15.24 & 29.57 & $\begin{array}{l}4.201 \\
4.632\end{array}$ & 49.80 & 3.257 \\
\hline 307.24 & $\begin{array}{l}15.24 \\
15.40\end{array}$ & 29.77 & $\begin{array}{l}4.032 \\
5.136\end{array}$ & 55.01 & 3.660 \\
\hline 315.07 & $\begin{array}{l}15.40 \\
15.54\end{array}$ & 29.96 & $\begin{array}{l}5.130 \\
5.713\end{array}$ & 57.86 & 3.822 \\
\hline 322.56 & 15.71 & 30.12 & $\begin{array}{l}0.713 \\
6.508\end{array}$ & 62.99 & 4.201 \\
\hline 330.55 & 15.87 & 30.26 & $\begin{array}{l}0.508 \\
8.177\end{array}$ & 68.42 & 4.585 \\
\hline 338.47 & 16.02 & 30.36 & 17.07 & 79.71 & 5.399 \\
\hline 346.14 & 16.17 & 30.41 & 78.38 & \multicolumn{2}{|c|}{ Enthalpy Run C } \\
\hline & & 30.42 & 167.66 & \multirow{2}{*}{\multicolumn{2}{|c|}{ Enthalpy Run D }} \\
\hline & & 30.421 & 187.79 & & \\
\hline & & 30.425 & 324.98 & \multirow{2}{*}{\multicolumn{2}{|c|}{ Series V }} \\
\hline & & 30.428 & 326.98 & & \\
\hline \multicolumn{2}{|c|}{ Series II } & 30.432 & 371.41 & 149.24 & 13.065 \\
\hline 5.70 & 0.0077 & 30.435 & 388.51 & 155.83 & 13.493 \\
\hline 6.45 & 0.0116 & 30.439 & 390.64 & 173.80 & 14.521 \\
\hline 7.16 & 0.0170 & 30.442 & 218.70 & 181.65 & 14.954 \\
\hline
\end{tabular}




\section{Results}

The heat capacity determinations expressed in terms of the thermochemical calorie, defined as $4.184 \mathrm{~J}$, an ice point of $273.15 \mathrm{~K}$, and the atomic weight of the natural uranium as 238.03 (one mole of $\mathrm{UO}_{2}$ has mass $270.07 \mathrm{~g}$ ) are listed in table 1 in chronological order. The heat capacity values are considered to have a probable error of about 5 per cent at $5 \mathrm{~K}, 1$ per cent at $10 \mathrm{~K}$, and less than 0.06 per cent above $25 \mathrm{~K}$, except in the peak region where very small $\Delta T$ 's were employed. These results have been adjusted for curvature, i.e. for the difference between the measured $\Delta H / \Delta T$ and the limit of the corresponding derivative. A close approximation to the values of

TABLE 2. Thermodynamic functions for uranium dioxide

\begin{tabular}{|c|c|c|c|c|}
\hline$\frac{T}{\mathrm{~K}}$ & $\frac{C_{p}}{\mathrm{cal} \mathrm{K}^{-1} \mathrm{~mol}^{-1}}$ & 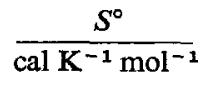 & $\frac{\left(H^{\circ}-H_{0}^{\circ}\right)}{\mathrm{cal} \mathrm{mol}^{-1}}$ & $\frac{-\left(G^{\circ}-H_{0}^{\circ}\right) / T}{\text { cal } \mathbf{K}^{-1} \mathrm{~mol}^{-1}}$ \\
\hline 5 & 0.0059 & 0.0017 & 0.006 & 0.0004 \\
\hline 10 & 0.0636 & 0.0173 & 0.134 & 0.0038 \\
\hline 15 & 0.277 & 0.0756 & 0.889 & 0.0164 \\
\hline 20 & 0.807 & 0.219 & 3.442 & 0.0468 \\
\hline 25 & 1.84 & 0.497 & 9.769 & 0.106 \\
\hline 30 & $(6.0)$ & - & - & - \\
\hline 35 & 2.37 & 1.953 & 53.38 & 0.428 \\
\hline 40 & 2.63 & 2.285 & 65.84 & 0.639 \\
\hline 45 & 2.93 & 2.612 & 79.72 & 0.840 \\
\hline 50 & 3.27 & 2.938 & 95.22 & 1.034 \\
\hline 60 & 3.98 & 3.597 & 131.5 & 1.406 \\
\hline 70 & 4.71 & 4.265 & 174.9 & 1.767 \\
\hline 80 & 5.43 & 4.941 & 225.6 & 2.121 \\
\hline 90 & 6.14 & 5.622 & 283.5 & 2.472 \\
\hline 100 & 6.83 & 6.304 & 348.3 & 2.821 \\
\hline 110 & 7.51 & 6.987 & 420.0 & 3.169 \\
\hline 120 & 8.17 & 7.669 & 498.4 & 3.516 \\
\hline 130 & 8.80 & 8.348 & 583.2 & 3.861 \\
\hline 140 & 9.41 & 9.023 & 674.4 & 4.206 \\
\hline 150 & 9.98 & 9.692 & 771.3 & 4.549 \\
\hline 160 & 10.52 & 10.353 & 873.9 & 4.892 \\
\hline 170 & 11.02 & 11.006 & 981.7 & 5.232 \\
\hline 180 & 11.49 & 11.650 & 1094.2 & 5.571 \\
\hline 190 & 11.92 & 12.283 & 1211.3 & 5.907 \\
\hline 200 & 12.32 & 12.904 & 1332.5 & 6.242 \\
\hline 210 & 12.70 & 13.515 & 1457.7 & 6.574 \\
\hline 220 & 13.05 & 14.114 & 1586.4 & 6.903 \\
\hline 230 & 13.38 & 14.701 & 1718.5 & 7.229 \\
\hline 240 & 13.69 & 15.277 & 1853.9 & 7.552 \\
\hline 250 & 13.99 & 15.842 & 1992.3 & 7.873 \\
\hline 260 & 14.27 & 16.396 & 2133.6 & 8.190 \\
\hline 270 & 14.53 & 16.940 & 2277.6 & 8.504 \\
\hline 280 & 14.79 & 17.473 & 2424.2 & 8.815 \\
\hline 290 & 15.02 & 17.996 & 2573.4 & 9.122 \\
\hline 300 & 15.24 & 18.509 & 2724.5 & 9.427 \\
\hline 350 & 16.23 & 20.934 & 3512.1 & 10.900 \\
\hline 273.15 & 14.61 & 17.11 & 2324.0 & 8.60 \\
\hline 298.15 & 15.20 & 18.41 & 2696.0 & 9.37 \\
\hline
\end{tabular}


$\Delta T$ used in the determinations usually can be estimated from the increments between adjacent mean temperatures shown in table 1.

The heat capacities at constant pressure $C_{p}$ and the derived thermodynamic functions $S^{\circ},\left(H^{\circ}-H_{0}^{\circ}\right)$, and $-\left(G^{\circ}-H_{0}^{\circ}\right) / T$ at selected temperatures presented in table 2 were obtained from the experimental thermal capacities by a least-squaresfitted curve through the experimental points (carefully compared with a large scale plot of the results) and the integration thereof. Both operations were performed by high-speed digital computers using programs previously described. Values below $5 \mathrm{~K}$ were extrapolated with a Debye $T^{3}$ heat capacity function. The thermodynamic functions are considered to have a precision indicated by a probable error of less than 0.1 per cent above $100 \mathrm{~K}$. An additional digit beyond those significant is given in table 2 for internal consistency and to permit interpolation and differentiation. Entropies and Gibbs energies have not been adjusted for nuclear spin and isotope mixing contributions and are thus practical values for use in chemical thermodynamic calculations.

\section{Discussion}

\section{COMPARISON WITH MAGNETIC, NEUTRON, AND OTHER THERMAL DATA}

Our results are shown in graphical form in figure 1 . The maximum in the heat capacity occurs at $30.44 \mathrm{~K}$, which can be compared with Néel temperatures of $30.80 \mathrm{~K}^{(8)}$ and $30.6 \mathrm{~K}^{(9)}$ determined by neutron elastic scattering experiments. The Néel temperature of $(30.5 \pm 0.5) \mathrm{K}$ reported by Cowley and Dolling ${ }^{(16)}$ from inelastic neutron scattering measurements also agrees well with our results. Magnetic susceptibility measurements taken in this laboratory by $\operatorname{Lin}^{(26)}$ on our heat-capacity sample show a peak in the susceptibility at $31.1 \mathrm{~K}$ and a maximum (nearly infinite) slope of the $\chi T$ against $T$ plot at $30.5 \mathrm{~K}$. A plot of the magnetic susceptibility results of Leask et al. (reference 5, figure 2) for $\mathrm{UO}_{2}$ was considered by these authors to show a maximum slope: “. . the temperature corresponding to $[\partial(\chi T) / \partial T]_{\max }, \approx 29 \mathrm{~K}$, agrees very well with the temperature of the $\lambda$-anomaly in the $\mathrm{UO}_{2}$ heat capacity which Jones et al. found at $28.5 \mathrm{~K}$ ". A replot of these values of $\chi T$ and $T$ (and even better a more accurate larger scale plot of their original results ${ }^{(27)}$ ), shows very clearly that the maximum slope occurs between 30.2 and $30.6 \mathrm{~K}$. For a simple antiferromagnet (i.e., where the ordered state is a result of exchange interactions), Fisher ${ }^{(28)}$ had identified the temperature of maximum slope in the $\chi_{\|} T$ function as the thermodynamic Néel temperature. As will be discussed later, $\mathrm{UO}_{2}$ is a somewhat more complicated substance than described by Fisher-the complication arising from the unusual lattice behavior near $T_{\mathrm{N}}$. Thus it is not clear whether Fisher's theory is applicable to $\mathrm{UO}_{2}$. Despite this difficulty in interpreting the susceptibility results, it is clear that they are not in conflict with the heat capacity results. Therefore it seems reasonable to accept $30.44 \mathrm{~K}$ as the thermodynamic transition temperature. The sharpness and height $\left(\approx 400 \mathrm{cal} \mathrm{mol}^{-1} \mathrm{~K}^{-1}\right)$ of our heat capacity peak (see figure 2) argue for a cooperative, essentially first-order component with a considerable portion of the enthalpy developed in a non-isothermal premonitory region. Con- 


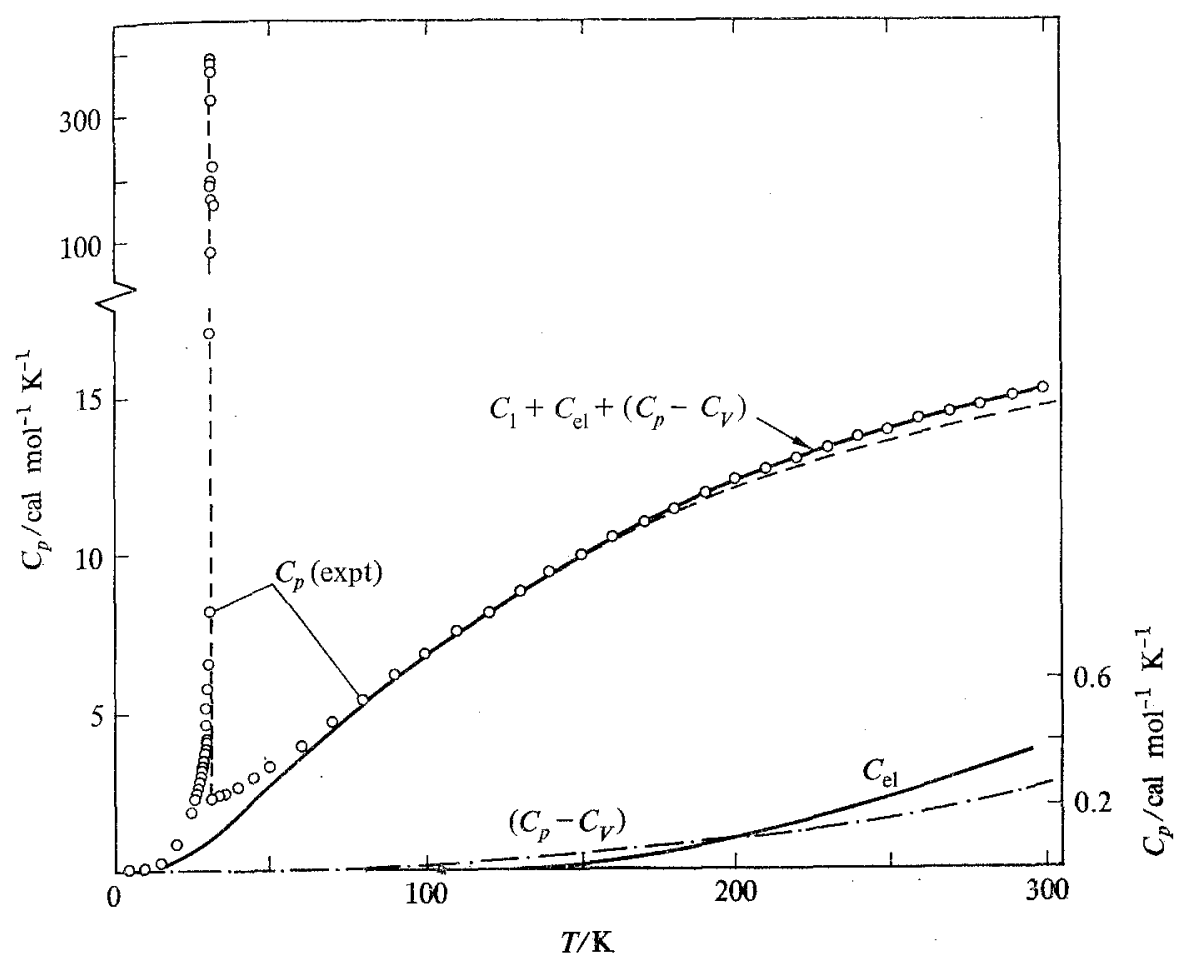

FIGURE 1. A plot of $C_{p}$ against $T$ for $\mathrm{UO}_{2}$. The solid circles represent the smoothed results $C_{p}$ (expt) everywhere except in the transition region. Here the points are the actual experimental ones. The symbols $C_{p}$ (calc.), $C_{1}, C_{\mathrm{el}}$, and $\left(C_{p}-C_{V}\right)$ are explained in the text. (Note that $C_{\mathrm{el}}$ and $\left(C_{p}-C_{V}\right)$ are referred to the right-hand scale which is magnified by a factor of 10.) The dotted line in the transition region serves only to connect the points.

sideration of the combined effects of departures from stoichiometry, chemical and phase purity, as well as crystallite size suggests reservation on this point until macroscopic single crystal material has been studied as a function of the uranium to oxygen mole ratio.

In figure 2 we compare our results with those of Jones et al. ${ }^{(1)}$ in the transition region. The differences between the two sets of measurements are immediately apparent. Not only are the shapes of the transition quite different, but the maximum in the heat capacity lies $1.7 \mathrm{~K}$ higher in the present results. These differences can probably be attributed to the fact that the measurements of Jones et al. were made on a sample of uncertain phase composition. They reported that their sample consisted of 99.3 moles per cent of $\mathrm{UO}_{2}$ and 0.7 moles per cent of $\mathrm{UO}_{3}$, but subsequent determinations of the phase relations ${ }^{(23)}$ in the uranium oxide system indicate that this sample was probably either $\mathrm{UO}_{2.01}$ if quenched or $\mathrm{UO}_{2}$ plus about 3 moles per cent of $\mathrm{U}_{4} \mathrm{O}_{9}$ if carefully annealed. The magnetic behavior of the $\mathrm{UO}_{2+x}$ phase (or of a mixture of $\mathrm{UO}_{2}$ and $\mathrm{U}_{4} \mathrm{O}_{9}$ phases) is not well understood although some information can be gained from the magnetic susceptibility measurements of Leask et al. ${ }^{(5)}$ 


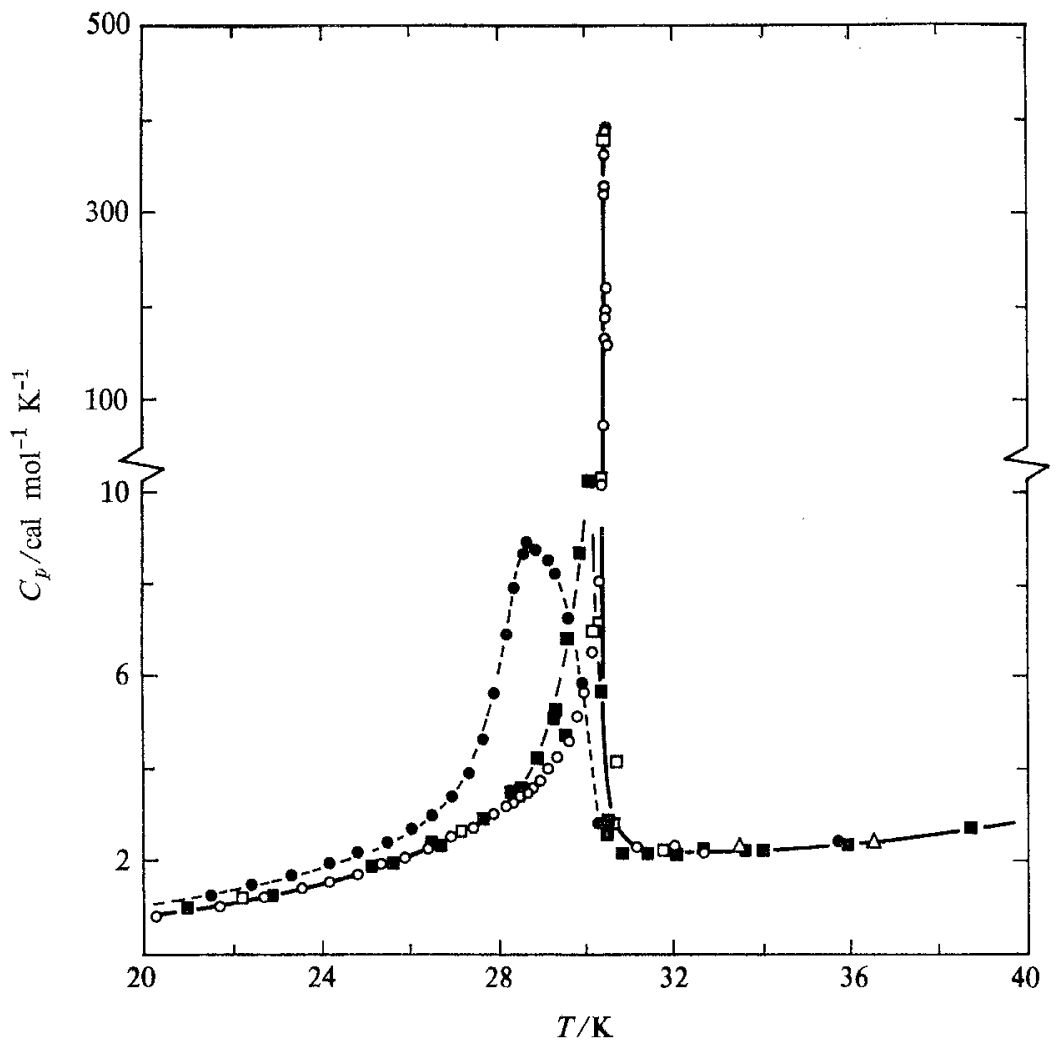

FIGURE 2. Heat capacity $C_{p}$ in the transition region. The solid line and $O, \square, \triangle$ refer to Series 1 , 2 , and 3 measurements on the hot-pressed $\mathrm{UO}_{2}$ sample. The long dashes and the $\square$ refer to measurements on the flame-fused sample. The short dashes and the are the results of Jones et $^{-.^{(1)}}$ on a less pure sample.

Their samples varied from a somewhat poorly defined $\mathrm{UO}_{2.098}$, which they thought to be cubic and similar to the high temperature $\mathrm{UO}_{2+x}$ phase, to a well defined sample of $\mathrm{UO}_{2.122}$ consisting of $\mathrm{UO}_{2}$ and $\mathrm{U}_{4} \mathrm{O}_{9}$ phases. For all the samples a susceptibility maximum was found at $6.4 \mathrm{~K}$. A susceptibility maximum at $6.4 \mathrm{~K}$ was also observed for $\mathrm{U}_{4} \mathrm{O}_{9}$-again with no indication of anomalous behaviour near $30 \mathrm{~K}$. Moreover, heat capacity measurements on $\mathrm{U}_{4} \mathrm{O}_{9}{ }^{(21,29)}$ show no evidence for a transition of any sort in the temperature range 1.6 to $300 \mathrm{~K}$. Thus it seems probable that even small departures from stoichiometry will have drastic effects on the magnetic properties of " $\mathrm{UO}_{2}$ " and may well explain the difference in the two heat capacity measurements.

Our heat capacity results above $300 \mathrm{~K}$ are in excellent agreement with the recent precise high-temperature results of Grønvold et al. ${ }^{(30)}$ by adiabatic calorimetry and of Fredrickson and Chasanov ${ }^{(31)}$ by modein enthalpy increment (drop calorimetric, method-of-mixtures) determination, but in significant disagreement with the results of Engel ${ }^{(32)}$ and of Moore and Kelley. ${ }^{(33)}$ For example, at $300 \mathrm{~K}$ we find $C_{P}=15.24$ cal mol ${ }^{-1} \mathrm{~K}^{-1}$. Grønvold et al. report 15.25 (for $\mathrm{UO}_{2.017}$ ), Fredrickson and Chasanov 15.25, Engel 16.80, and Moore and Kelley $15.62 \mathrm{cal} \mathrm{mol}^{-1} \mathrm{~K}^{-1}$. 


\section{RESOLUTION OF THE HEAT CAPACITY}

General considerations. Various schemes have been employed to achieve a satisfactory estimation of the lattice contributions to the heat capacity in uranium and other actinide dioxides. Jones et al. ${ }^{(1)}$ used a simple graphical interpolation method to estimate this contribution in the immediate vicinity of the transition region. Combinations of Debye and Einstein functions selected on the basis of a modified Lindemann equation (relating the characteristic temperatures to such parameters as the melting temperature, molar volume, molar mass, etc.) have been used by Smith. ${ }^{(34)}$ The thermal capacity of isostructural diamagnetic thorium dioxide was used by Osborne et al..$^{(35)}$ to estimate the lattice contribution in uranium dioxide. The applicability of this method is reinforced by comparison of Debye characteristic temperatures evaluated from infrared dielectric dispersion results on both $\mathrm{ThO}_{2}$ and $\mathrm{UO}_{2},{ }^{(36)}$ from thermal conductivities, ${ }^{(37)}$ and from elastic constants. ${ }^{(38)}$ The corresponding states approach of Stout, ${ }^{(39)}$ however, seems not to have been employed. The $\mathrm{UO}_{2}$ Debye-Waller factors, from which values of $\Theta_{D}{ }^{(7)}$ and therefore the lattice contributions to $C_{V}$ can be derived, have been measured by Willis ${ }^{(40)}$ in the temperature range 293 to $1400 \mathrm{~K}$. These values, however, are not appropriate for temperatures below $293 \mathrm{~K}$ because of the temperature dependence of $\Theta_{\mathrm{D}}$. Finally, the inelastic neutron scattering work of Dolling et al., ${ }^{(14)}$ in which the phonon dispersion relations at $296 \mathrm{~K}$ were measured, provides perhaps the best possibility for resolving the several contributions to the heat capacity. The mode frequencies are confirmed by infrared reflectivities. Using an appropriate interionic force model to fit the experimental dispersion relations, they derived the phonon density of states and the harmonic lattice contribution to $C_{V}$ in the temperature range 1 to $500 \mathrm{~K}$. We have predicated much of our subsequent discussion on these results.

Above the Néel temperature. It is to be expected that lattice vibrations will constitute the major part of the heat capacity above $30 \mathrm{~K}$. For this we take the results of Dolling et al. ${ }^{(14)}$ described above. In addition to the harmonic contribution of the normal modes, there will also be an anharmonic contribution, which implies a difference between $C_{p}$ and $C_{V}:\left(C_{p}-C_{V}\right)=\left(x^{2} / \kappa\right) V_{\mathrm{m}} T$ where $\alpha$ is the thermal expansivity, $\kappa$ the isothermal compressibility, and $V_{\mathrm{m}}$ the molar volume. The $\left(C_{p}-C_{V}\right)$ contribution may be approximated with the Grüneisen parameter, i.e., $\left(C_{p}-C_{V}\right)=\alpha \Gamma T C_{V}$ in which $\Gamma=\alpha V_{\mathrm{m}} / \kappa C_{V}$. In the analysis of their high temperature results Grønvold et al. ${ }^{(30)}$ calculated $\Gamma=2.1$ and used the harmonic contribution to $C_{V}$ as calculated by Dolling et al. ${ }^{(14)}$ to determine $\left(C_{p}-C_{V}\right)$. In our analysis we use the same values of $\Gamma$ and $C_{V}$ and take $\alpha=25.4 \times 10^{-6} \mathrm{~K}^{-1}$ at $298 \mathrm{~K}$ which is the average of X-ray ${ }^{(41)}$ and bulk expansion data ${ }^{(42)}$ and proportional to $C_{p}$ at lower temperatures. Finally there is a small electronic contribution resulting from higher crystal-field states. Their associated energies and degeneracies have been calculated by Rahman and Runciman ${ }^{(43)}$ on the basis of a point charge calculation. Grønvold et al. ${ }^{(30)}$ however found better agreement with their heat capacity results by retaining the order of the various levels but re-adjusting the energies. The energies of the first four levels are given in table 3. Using those of Grønvold et al., we have evaluated the electronic heat capacity contribution. At $300 \mathrm{~K}$ this amounts to $0.38 \mathrm{cal} \mathrm{mol}^{-1} \mathrm{~K}^{-1}$. 
TABLE 3. The wavenumbers $\tilde{v}$ of the first four levels in the crystal-field treatment

\begin{tabular}{ccc}
\hline Level & \multicolumn{2}{c}{$\tilde{v} / \mathrm{cm}^{-1}$} \\
& Rahman and Runciman ${ }^{(43)}$ & Grønvold et al. ${ }^{(30)}$ \\
\hline$T_{2}$ & 0 & 0 \\
$E$ & 1368 & 900 \\
$T_{1}$ & 5034 & 1600 \\
$A_{1}$ & 5726 & 2900 \\
\hline
\end{tabular}

In figure 1 the various components of the heat capacity, harmonic lattice $C_{1}$ as determined by Dolling et al., electronic $C_{\mathrm{el}}$, and anharmonic $\left(C_{p}-C_{V}\right)$, are shown. The solid line labelled $C_{p}$ (calc.) represents the sum: $\left\{C_{1}+C_{\mathrm{el}}+\left(C_{p}-C_{V}\right)\right\}$. Above $120 \mathrm{~K}$ the agreement between $C_{p}\left(\right.$ expt) and $C_{p}$ (calc.) is excellent, the discrepancy being less than 1 per cent. The excess heat capacity, $C_{\text {ex }}=\left\{C_{p}(\operatorname{expt})-C_{p}(\mathrm{calc}).\right\}$, is plotted in figure 3 . Above $200 \mathrm{~K}, C_{p}$ (calc.) exceeds the measured values by a small amount. This undoubtedly arises, in part, from over-estimating $C_{\mathrm{el}}$, but until accurate spectroscopic values of the crystal-field energies are available no firm conclusion can be drawn. Uncertainty in the dispersion calculations and about anharmonic contributions other than $C_{\mathrm{di}}$ may also be of importance.

The most interesting feature of figure 3 , however, is the high excess heat capacity in the temperature region 30 to $120 \mathrm{~K}$. This was previously noted by Dolling et al. ${ }^{(14)}$ who suggested considerable short-range ordering above $T_{\mathrm{N}}$ as the apparent cause. The elastic constants of Brandt and Walker ${ }^{(10,11)}$ and the thermal conductivities of Aring and Sievers ${ }^{(13)}$ and Moore and McElroy ${ }^{(37)}$ indicate, however, that the

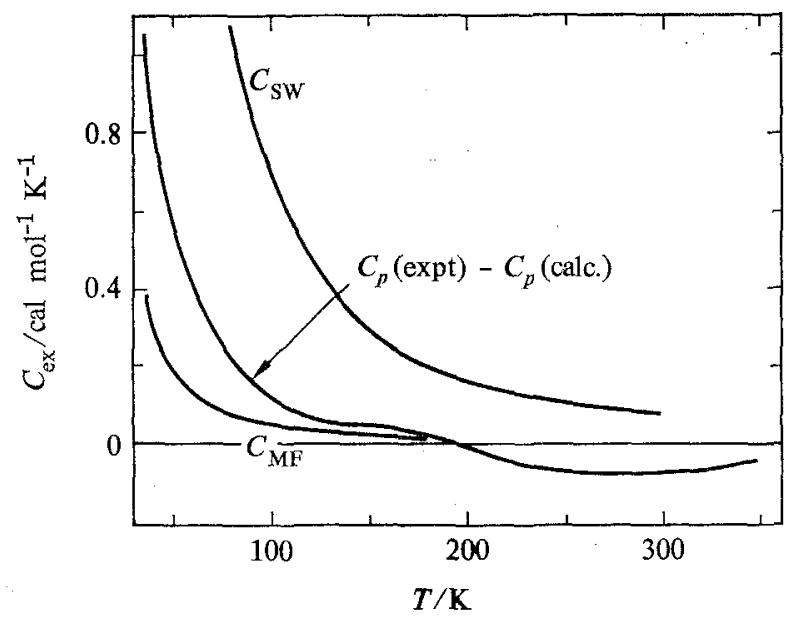

FIGURE 3. Excess heat capacity above $T_{\mathrm{N}}$. The curves labelled $C_{\mathrm{MF}}$ and $C_{\mathrm{Sw}}$ are theoretical estimates of the excess (magnetic) heat capacity derived from Allen's molecular-field and spin-wave formalisms, respectively. The curve labelled $\left\{C_{p}(\operatorname{expt})-C_{p}(\right.$ calc. $\left.)\right\}\left(=C_{e x}\right)$ is the heat capacity remaining when the lattice, electronic and $\left(C_{p}-C_{V}\right)$ contributions have been subtracted. 
situation may be considerably more complex. The broad anomalies in the lattice properties, which extend well above $T_{\mathrm{N}}$, contrast with the sharpness of the magnetization curve for antiferromagnetic $\mathrm{UO}_{2}$.

In an attempt to explain the behavior of $\mathrm{UO}_{2}, \mathrm{Allen}^{(44,45)}$ has constructed a theoretical model in which he considered the exchange interaction between $\mathrm{U}^{4+}$ ions, the elastic energy of the lattice, and the spin-lattice interaction (which he described in terms of the Jahn-Teller effect). The antiferromagnetic, spin-lattice ground state was derived in the molecular field approach by minimizing the suitably parameterized energy and was found to be incompletely polarized because of a competition between exchange and Jahn-Teller forces. That is, the ground state is characterized not only by a net spin but also by a nonzero average quadrupole moment for the $\mathrm{U}^{4+}$ ions. Using this ground state, Allen then determined the values of the parameters in two separate approaches. In the first method ${ }^{(44)}$ his theory was fitted to the antiferromagnetic resonances observed in both his far-infrared work ${ }^{(44)}$ and in the inelastic neutron scattering results of Dolling and Cowley. ${ }^{(16)}$ To account for the resonances, he reformulated the magnetic interactions in terms of spin-wave theory and also considered indirect quadrupole-quadrupole effects, which he found to be large. Both of these interactions have nonzero, off-diagonal matrix elements which can generate the antiferromagnetic excitations. For our purposes we shall label this the spin-wave (SW) case. In the second approach Allen ${ }^{(45)}$ treated the $^{2}$ magnetic interactions completely in the molecular field framework and derived an expression for the Gibbs energy $G$ which contained the above mentioned parameters, the average spin, and the average quadrupole moment. Above $T_{\mathrm{N}}$ the spin and quadrupole effects in the molecular field approximation vanish and a relatively simple form for $G$ was obtained. This could be compared with experimental results since the elastic constant $c_{44}=\mathrm{d}^{2} G / \mathrm{d} U_{4}^{2}$ (in which $U_{4}$ is an external strain). Thus the parameters were obtained by normalizing $c_{44}$ (theoretical) to the experimental values of Brandt and Walker ${ }^{(10,11)}$ at $240 \mathrm{~K}$. We label this approach the molecularfield (MF) case.

With the Gibbs function derived by Allen, it is possible to compare the theory with our observed excess heat capacity above $T_{\mathrm{N}}$ since $C_{p}=\left(\partial^{2} G / \partial T^{2}\right)_{p}$. We do this in two ways. We first use the molecular-field parameters to calculate the excess heat capacity $C_{\mathrm{MF}}$ from the molecular-field Gibbs function. Secondly, we use the spin-wave parameters to calculate another excess heat capacity $C_{\mathrm{SW}}$, again using the molecular-field Gibbs function. This is a valid approach since above $T_{\mathrm{N}}$ the Gibbs function depends only on parameters which are common to both the spin-wave and molecular-field formalisms. These two heat capacities are compared with our excess heat capacity $C_{\mathrm{ex}}$ in figure 3 , where it is seen that $C_{\mathrm{MF}}$ lies somewhat below $C_{\mathrm{ex}}$ while $C_{\mathrm{Sw}}$ lies considerably above $C_{\mathrm{ex}}$. Because magnetic interactions, and, in particular, short-range magnetic ordering effects are neglected in the molecular-field Gibbs function, it is expected that the derived heat capacity would be less than that observed. This is the case for $C_{\mathrm{MF}}$. The rather large discrepancy between $C_{\mathrm{Sw}}$ and $C_{\mathrm{MF}}$, however, indicates a lack of consistency between Allen's two formalisms and may suggest a problem in describing the $\mathrm{UO}_{2}$ spin-lattice interaction in terms of a Jahn-Teller effect. In fairness, however, it should be mentioned that we are com- 
paring our results with the second derivative of Allen's Gibbs function-a procedure which invariably exaggerates discrepancies. Moreover, Allen himself claims only qualitative agreement between theory and experiment.

Brandt and Walker ${ }^{(11)}$ have also considered the role of the Jahn-Teller effect in the analysis of their elastic constant and ultrasonic attenuation results for $\mathrm{UO}_{2}$. They explained the behavior of $c_{44}$ in terms of a coupling between a $\mathrm{U}^{4+}$ ion and lattice strains and examined the possibility that this coupling arose from Jahn-Teller interactions. Their analysis suggested that Jahn-Teller coupling was not appropriate, but because of the necessarily qualitative nature of their treatment they neither accepted nor rejected this type of coupling. In addition they attributed the behavior of $c_{11},\left(c_{11}-c_{12}\right)$, and $c_{44}$ near $T_{\mathrm{N}}$ to magnetic exchange interactions. Blume ${ }^{(46)}$ has also developed a theory of the first-order transition in $\mathrm{UO}_{2}$ which is critically dependent upon a singlet crystal field ground state of the $\mathrm{U}^{4+}$ ion. Because the farinfrared work of Allen, ${ }^{(44)}$ the susceptibility work of Comly, ${ }^{(47)}$ and the crystal field calculations of Rahman and Runciman ${ }^{(43)}$ all indicate that the ground state is a triplet, Blume's theory is probably not applicable to $\mathrm{UO}_{2}$.

Hence in the temperature region 120 to $350 \mathrm{~K}$ the thermal behavior of $\mathrm{UO}_{2}$ is well understood. In the paramagnetic region between the Néel temperature and $120 \mathrm{~K}$ there is a significant excess heat capacity which, though it is not quantitatively explained by existing theory, involves coupling between the triplet ground state and the lattice as well as short-range ordering effects via the exchange interaction.

Below the Néel temperature. The magnon dispersion relations have been studied by Cowley and Dolling ${ }^{(15,16,18)}$ using inelastic neutron scattering techniques. They also observed a significant alteration in the phonon dispersion relations below $T_{\mathrm{N}}$ and attributed this to a strong magnon-phonon interaction in the antiferromagnetic phase. To fit their experimental dispersion relations, they constructed several spinwave models which took into account nearest-neighbor and next-nearest-neighbor exchange and spin anisotropy. The magnon-phonon coupling, however was not explicitly considered. Using the best of these models (BB), Cracknell and Joshua ${ }^{(17)}$ calculated the magnon contribution for various temperatures below $20 \mathrm{~K}$. An earlier calculation of this contribution by De Batist et al. ${ }^{(48)}$ used a combination spin-wave, molecular field Hamiltonian, which was parameterized with two exchange integrals (nearest- and next-nearest-neighbor exchange) and a fictitious molecular field. Using cylindrically symmetric dispersion relations, they derived a parameterized magnetic heat capacity. The parameters were determined by subtracting the phonon contribution using $\Theta_{\mathrm{D}}=270 \mathrm{~K}$ (calculated from room temperature elastic constants) from the heat capacity results of Jones et al..$^{(1)}$ and comparing the resultant excess heat capacity with their derived heat capacity. We have chosen, however, to base our subsequent discussion on the results of Cracknell and Joshua because they are more firmly based on experimental results and make no assumptions regarding the symmetry of the dispersion relations.

In figure 4 we compare our experimental results below $T_{\mathrm{N}}$ with those derived from the neutron work. The agreement is only fair, the discrepancy being greater than 15 per cent at $15 \mathrm{~K}$. For several reasons, however, the agreement is probably 


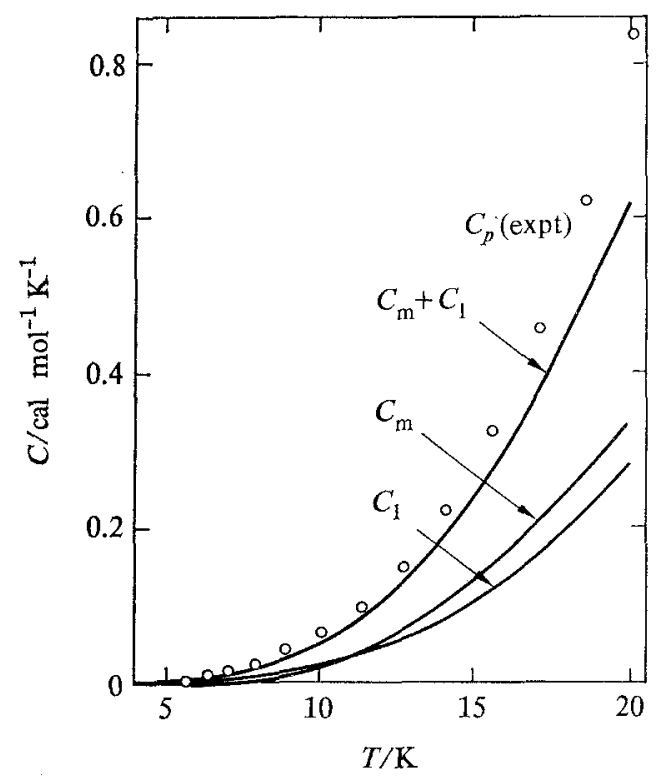

FIGURE 4. Excess heat capacity below $T_{N}$. The $\bigcirc$ are actual experimental results. The curves labelled $C_{\mathrm{m}}$ and $C_{1}$ represent the magnetic and lattice contributions respectively as determined by inelastic neutron scattering.

as good as can be expected. First, as Cowley and Dolling ${ }^{(16)}$ point out, the coupled magnon-phonon dispersion relations should be used to calculate the density of states. Although this calculation has not been done, it seems likely that the neglect of coupling could account for most of the discrepancy. Second, because of the multidomain structure of antiferromagnetic $\mathrm{UO}_{2}$, it is difficult to determine the exchange and anisotropy constants. Thus there may be some error associated with the model parameters themselves. Finally, the model does not predict the Néel temperature and the Curie-Weiss constant correctly and violates one of the three stability criteria. Since the model is basically only an interpolation device to compute the density of states, the fact that the Néel temperature, for example, is incorrectly predicted may not be a serious indictment.

A plot of $C_{p} / T$ against $T^{2}$ for the data in table 1 up to $10 \mathrm{~K}$ is shown in figure 5 together with an extrapolated curve for $\mathrm{ThO}_{2}$ calculated from the Debye heat capacity function with $\Theta_{\mathrm{D}}=253 \mathrm{~K}$, which is based upon thermal data extending down to $10 \mathrm{~K}$. It is interesting to note that $\mathrm{UO}_{2.250},{ }^{(28)} \alpha, \beta$, and $\gamma-\mathrm{UO}_{2.333},{ }^{(49)}$ and $\mathrm{UO}_{2.667},{ }^{(50)}$ with similar structures, the same ion masses, and nearly the same lattice constants have much greater heat capacities in this region.

Thermodynamics of the magnetic transition. The entropy associated with a magnetic transition can often provide useful information about the nature of the crystal field states involved. This entropy is usually evaluated by subtracting the lattice contribution from the measured entropy. $\mathrm{UO}_{2}$, however, presents a somewhat more 


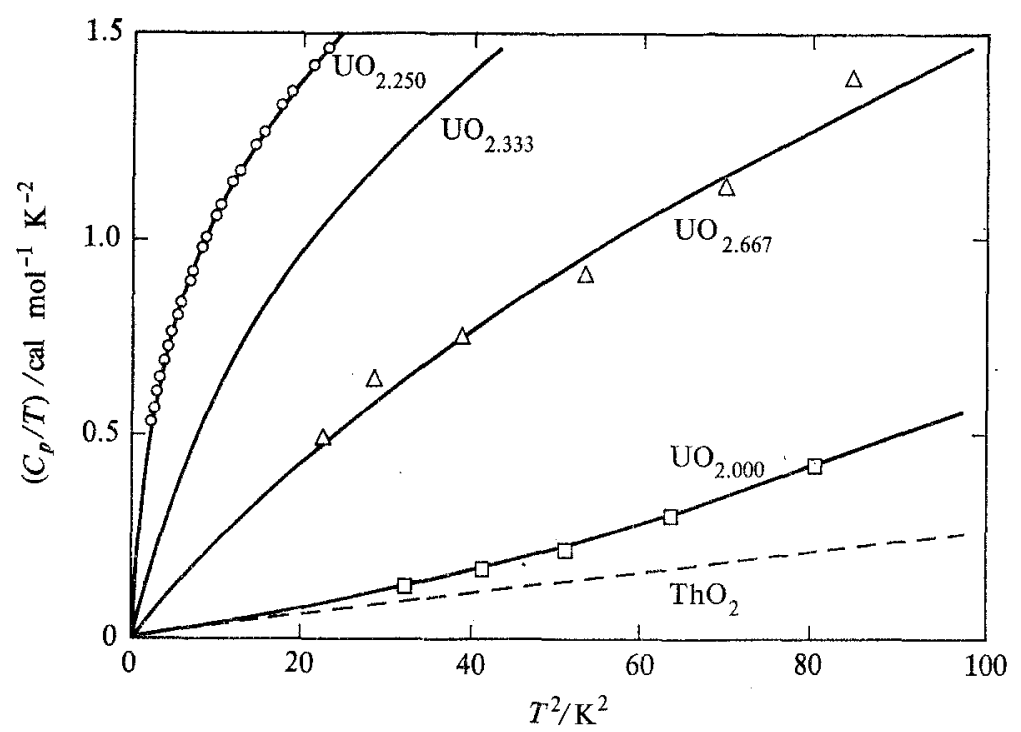

FIGURE 5. A plot of $C_{p} / T$ against $T^{2}$ for certain actinide oxides. The $\mathrm{UO}_{2}$ values are from the present work; sources for the other data are described in the text.

complex situation because of the coupled magnetic-lattice character of the transition. Thus if we subtract the contribution of the vibrational normal modes, we obtain a transitional entropy with both lattice and magnetic contributions. Because a further separation is not possible, we can give only an upper limit for the "magnetic" entropy associated with the $\mathrm{UO}_{2}$ crystal field ground state. If we utilize the lattice heat capacity estimate based upon the inelastic neutron scattering measurements of Dolling et al. ${ }^{(14)}$ together with the electronic and anharmonic corrections shown in figure 1, the temperature dependence of the resulting $S_{\mathrm{ex}}$ is as depicted in figure 6 . At the Néel temperature an entropy contribution of only about $1.5 \mathrm{cal} \mathrm{mol}^{-1} \mathrm{~K}^{-1}$ has been developed. At $120 \mathrm{~K}$, where the excess heat capacity is small and the contribution from higher crystal field states should be negligible, we find an excess entropy of $2.0 \mathrm{cal} \mathrm{mol}{ }^{-1} \mathrm{~K}^{-1}$. For a triplet ground state we expect an entropy increment of $R \ln 3=2.18 \mathrm{cal} \mathrm{mol}^{-1} \mathrm{~K}^{-1}$ which is in reasonable agreement with our experimental value.

As noted earlier, alternative methods for estimation of the lattice heat capacity of $\mathrm{UO}_{2}$ have been used. Smith's ${ }^{(34)}$ method of selecting Debye and Einstein characteristic temperatures for the $\mathrm{UO}_{2}$ lattice resulted in an overall estimate for $S_{\text {ex }}$ of 2.2 cal mol ${ }^{-1} \mathrm{~K}^{-1}$. Use of Willis's ${ }^{(40)}$ characteristic temperatures based on DebyeWaller factors for $\mathrm{UO}_{2}$ and $\mathrm{ThO}_{2}$ gives a lattice contribution which exceeds (for reasons noted earlier) the experimental $C_{p}$ curve at the relatively low temperature of $45 \mathrm{~K}$. Results of both these approaches are also depicted in figure 6. Use of the measured $C_{p}$ of isostructural, diamagnetic $\mathrm{ThO}_{2}$ as the lattice contribution for $\mathrm{UO}_{2}$ gives a higher estimate of $S_{\mathrm{ex}}$, as also may be noted in figure 6 , than does any of the other methods. Between $35 \mathrm{~K}$ and $300 \mathrm{~K}$ this lattice heat capacity estimate is con- 


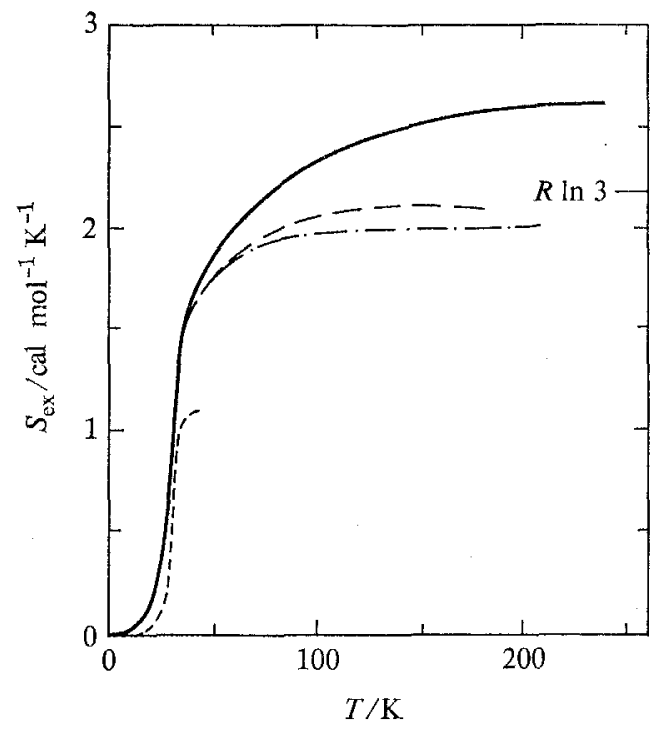

FIGURE 6. Excess entropy of $\mathrm{UO}_{2}$. The several curves of $S_{\text {ex }}$ extend to temperatures where the adjusted lattice heat capacity becomes tangential to (or intersects) $S$ (expt) for $\mathrm{UO}_{2}$. That indicated by . - is based on Dolling and Cowley's ${ }^{(14)}$ neutron scattering results (with adjustment for $C_{\text {el }}$ and $\left(C_{p}-C_{V}\right)$ ); —_ is based on the thermal capacity of $\mathrm{ThO}_{2}$ results; ${ }^{(35)}{ }_{-} \ldots \ldots$, by Smith's method; ${ }^{(34)}$

sistently between 0.15 and $0.45 \mathrm{cal} \mathrm{mol}^{-1} \mathrm{~K}^{-1}$ lower than that from the neutron result. $\mathrm{ThO}_{2}$ would seem to be an unusually favorable isostructural "stand-in" for the reasons noted previously.

Although we have preferred the approach based on the neutron scattering results, figures 6 and 3 do temper somewhat pretensions as to the precision of both the essentially theoretical and the essentially experimental evaluations of the excess entropy.

We are grateful to the Division of Research of the United States Atomic Energy Commission for enabling financial support and to the National Science Foundation for research participation awards for one of us (J.J.H.). We would like to acknowledge the kind assistance of Dr Gerald Dolling (for a detailed tabulation of the heat capacity of $\mathrm{UO}_{2}$ derived from the neutron scattering results of reference 14), that of $\mathrm{Dr}$ Ludmila Michăelova Jakeš (who assisted in a preliminary endeavor to resolve the lattice heat capacity), that of Dr C. W. Kuhlman and the Mallinckrodt Chemical Works (for provision of the pressed and flame-fused calorimetric samples), and the interest and suggestions of Drs George H. Winslow and J. M. Leitnaker.

\section{REFERENCES}

1. Jones, W. M.; Gordon, J.; Long, E. A. J. Chem. Phys. 1952, 20, 695.

2. Dawson, J. K.; Lister, M. W. J. Chem. Soc. (London), 1950, 2181.

3. Henry, W. E. Phys. Rev. 1958, 109, 1976.

4. Arrott, A.; Goldman, J. E. Phys. Rev. 1957, 108, 948. 
5. Leask, M. J. M.; Roberts, L. E. J.; Walter, A. J.; Wolf, W. P. J. Chem. Soc. (London), 1963, 4788.

6. Henshaw, D. G.; Brockhouse, B. N. Bull. Amer. Phys. Soc. 1957, 2, 9.

7. I. A. E. A. Thermodynamics Panel, March 1964, reported in Thermodynamic and Transport Properties of Uranium Dioxide and Related Phases, Technical Report Series, No. 39. Holley C. E.; editor. International Atomic Energy Agency, Vienna. 1965, pp. 23 et seq.

8. Frazer, B. C.; Shirane, G.; Cox, D. E.; Olsen, C. E. Phys. Rev. 1965, 140, A1448.

9. Willis, B. T. M.; Taylor, R. I. Phys. Lett. 1965, 17, 188.

10. Brandt, O. G.; Walker, C. T. Phys. Rev. Lett. 1967, 18, 11.

11. Brandt, O. G.; Walker, C. T. Phys. Rev. 1968, 170, 528.

12. de Kouchkovsky, R.; Lecomte, M. C. R. H. Acad. Sci. B. 1968, 267, 620.

13. Aring, K.; Sievers, A. J. J. Appl. Phys. 1967, 38, 1496.

14. Dolling, G.; Cowley, R. A.; Woods, A. D. B. Can. J. Phys. 1965, 43, 1397.

15. Dolling, G.; Cowley, R. A. J. Appl. Phys. 1968, 39, 1111.

16. Cowley, R. A.; Dolling, G. Phys. Rev. 1968, 167, 464.

17. Cracknell, A. P.; Joshua, S. J. Phys. Status. Solidi 1969, 36, 737.

18. Dolling, G.; Cowley, R. A. Phys. Rev. Lett. 1966, 16, 683.

19. Westrum, E. F., Jr.; Grønvold, F. J. Amer. Chem. Soc. 1959, 81, 1777.

20. Westrum, E. F., Jr.; Grønvold, F. J. Phys. Chem. Solids 1962, $23,36$.

21. Osborne, D. W.; Westrum, E. F., Jr.; Lohr, H. R. J. Amer. Chem. Soc. 1957, 79, 529.

22. Westrum, E. F., Jr.; Grønvold, F. Thermodynamics of Nuclear Materials. International Atomic Energy Agency: Vienna. 1962.

23. Grønvold, F. J. Inorg. Nucl. Chem. 1955, 1, 357.

24. Westrum, E. F., Jr.; Furukawa, G. T.; McCullough, J. P. Adiabatic low-temperature calorimetry, In Experimental Thermodynamics, Vol. I. McCullough, J. P.; Scott, D. W.; editors. Butterworths: London. 1968.

25. For supplementary material including the detailed data on the flame-fused sample, magnetic data, and the resolution of the heat capacity data order NAPS Document 01180 from ASIS National Auxiliary Publications Service, c/o CCM Information Sciences, Inc., 909 Third Avenue, New York, New York, 10022 , remitting $\$ 5.00$ for microfiche or $\$ 2.00$ for photocopies.

26. Lin, M. S. Ph.D. Thesis, The University of Michigan, Ann Arbor, Michigan 1964, Dissertation Abstr. No. 65-5924.

27. Provided by the courtesy of W. P. Wolf, Personal communication to Edgar F. Westrum, Jr., Dec. 1963.

28. Fisher, M. E. Phil. Mag. (London), 1962, 7, 1731.

29. Flotow, H. E.; Osborne, D. W.; Westrum, E. F., Jr. J. Chem. Phys. 1968, 49, 2438.

30. Grønvold, F.; Kveseth, N. J.; Sveen, A.; Tichy, J. J. Chem. Thermodynamics 1970, 2, 665. .

31. Fredrickson, D. R.; Chasanov, M. G. J. Chem. Thermodynamics 1970, 2, 623.

32. Engel, T. K. J. Nucl. Mater. 1969, 31, 211.

33. Moore, G. E.; Kelley, K. K. J. Amer. Chem. Soc. 1947, 69, 2105.

34. Smith, D. Personal communication, 1970, cf. Smith, D. J. Phys. Chem. Solids 1968, 29, 525

35. Osborne, D. W.; Westrum, E. F., Jr. J. Chem. Phys. 1953, 21, 1884.

36. Axe, J. D.; Pettit, G. D. Phys. Rev. 1966, 151, 676.

37. Moore, J. P.; McElroy, D. L. J. Amer. Ceramic Soc. In Press.

38. Ali, M.; Nagels, P. Phys. Status Solidi 1967, 21, 113.

39. Stout, J. W.; Catalano, H. J. Chem. Phys. 1955, 23, 2013.

40. Willis, B. T. M. Proc. Roy. Soc. 1963, A274, 122 and 1963, A274, 134.

41. Kempter, C. P.; Elliott, R. O. J. Chem. Phys. 1959, 30, 1524.

42. Burdick, M. D.; Parker, H. S. J. Amer. Ceramic Soc. 1956, 39, 181.

43. Rahman, H. U.; Runciman, W. A. J. Phys. Chem. Solids 1966, 27, 1833.

44. Allen, S. J. Phys, Rev. 1968, 166, 530.

45. Allen, S. J. Phys. Rev. 1968, 167, 492.

46. Blume, M. Phys. Rev. 1966, 141, 517.

47. Comly, J. B. J. Appl. Phys. 1968, 39, 716.

48. De Batist, R.; Gevers, R.; Verschuren, M. Phys. Status Solidi 1967, 19, 77.

49. Westrum, E. F., Jr.; Cordfunke, E. H. P. unpublished data.

50. Westrum, E. F., Jr.; Grønvold, F. J. Amer. Chem. Soc. 1959, 81, 1777. 